\title{
Signatures of hypercharge axions at contemporary and future colliders
}

\author{
Ram Brustein $^{*}$ and Yael Raveh ${ }^{\dagger}$ \\ Department of Physics, Ben-Gurion University, Beer-Sheva 84105, Israel
}

(Received 28 April 2018; published 10 September 2018)

\begin{abstract}
We investigate the possible detection of the hypercharge axion (HCA) in colliders. The HCA is a hypothetical pseudoscalar that couples to weak hypercharge topological density and could potentially explain the dominance of matter over antimatter in the observable universe. If the HCA exists, it can be produced in colliders via vector boson fusion or in association with a photon or with a $Z$ boson, and detected by looking for its decay into photons or $Z$ bosons. We find that for certain values of the HCA mass and coupling scales, both of the order of a TeV, existing data from the Large Hadron Collider (LHC) can already put interesting constraints, and in future colliders, such as the High Luminosity LHC, the accessible detection range is increased significantly.
\end{abstract}

DOI: 10.1103/PhysRevD.98.055010

\section{INTRODUCTION}

Understanding how the baryon asymmetry of the Universe originated is one of the fundamental open questions in high energy physics and cosmology today. Currently, the prevailing theory is that the Universe started out as baryon symmetric, but now is not, due to some process that generated matter excess after the big bang [1].

One of the suggested baryogenesis mechanisms incorporates hyperelectric and hypermagnetic fields into electroweak baryogenesis [2-4]. It was observed that: (i) a topological number condensate can be released at the electroweak phase transition (EWPT) in the form of leptons and baryons, and (ii) strong enough hypermagnetic fields could make the EWPT strongly first order.

Scalars with axionlike coupling to hypercharge fields were previously considered in $[5,6]$. Amplification of ordinary electromagnetic fields by such scalar fields was discussed in [5] and their possible use for baryogenesis in [6].

More recently, in 2015, the ATLAS and CMS Collaborations at the Large Hadron Collider (LHC) reported preliminary data with a small excess of diphoton events at an invariant mass of about $750 \mathrm{GeV}$. Though the excess was absent in data collected during 2016 and is now considered a statistical fluctuation [7,8], it nonetheless triggered a lot of attention among the particle physics community; hundreds of theory papers appeared following

\footnotetext{
*ramyb@bgu.ac.il

†raveh.yl@gmail.com
}

Published by the American Physical Society under the terms of the Creative Commons Attribution 4.0 International license. Further distribution of this work must maintain attribution to the author(s) and the published article's title, journal citation, and DOI. Funded by SCOAP. the 2015 announcement [9]. Particularly, several theory papers suggested that if such a discovery was confirmed, it would require unexpected new elementary particles. One of the most widely studied explanations relied on a spin- 0 real singlet with effective interactions to the Standard Model (SM) gauge bosons (see, e.g., [10-13]).

The hypercharge axion (HCA) is a hypothetical pseudoscalar with electroweak interactions. It was first proposed in 1999 by Brustein and Oaknin [14] as a candidate for inducing baryogenesis; in cosmology, the HCA can exponentially amplify hypercharge fields in the symmetric phase of the electroweak plasma, while coherently rolling or oscillating [15], leading to the formation of a timedependent condensate of topological number density. This condensate can be converted at the EWPT, under certain conditions, into baryons in sufficient quantity to explain the observed baryon asymmetry in the Universe [16]. More recently, similar ideas were presented in $[17,18]$.

To be effective for baryogenesis, the HCA has to couple mainly to the topological density of the hypercharge fields. Hence the focus in this paper on this coupling. However, in general, it would have additional couplings to fermions and to $W$ bosons [19]. In [20], an extensive analysis was performed in which additional operators that appear as HCA interactions in more realistic extensions of the standard model were considered. In that paper it was noticed that the possible candidates usually do have such additional couplings.

In particular, the minimal supersymmetric standard model (MSSM) and four-dimensional (4D) string/M-theory low energy effective field theories were studied in [20], looking for suitable candidates for HCA which couple mostly to the hypercharge topological density. It was concluded that the MSSM does not contain an HCA that can successfully drive baryogenesis, because of the 
appearance of the additional couplings. In some specific models with broken $R$-parity, sneutrinos do couple to topological gauge densities through triangle diagrams but also fail to serve as HCAs due to chiral symmetry restoration in the unbroken phase of the electroeak (EW) theory.

It was also pointed out in [20] that stringy axions could couple directly to hypercharge topological density at the compactification scale, which is typically much higher than the EW scale. But, it was concluded that in generic compactifications the specific conditions for successful baryogenesis are violated in one way or another. More elaborate models which may lead to HCAs obey all the conditions. Such models may be realized in some special compactification schemes, or in scenarios of very low string scale.

In this paper, we will assume that the conditions that guarantee that the HCA couples mainly to hypercharge fields are satisfied, and therefore its coupling to additional operators is suppressed. Since the hypercharge photon is a linear combination of the ordinary photon and $Z$ boson, HCAs couple to photons and $Z$ bosons. As a result, the HCA can be produced in interactions involving photons and $Z$ bosons and detected by looking for its decay into photons or $Z$ bosons. For this reason, in addition to the fact that the HCA can be relevant to baryogenesis for a range of masses of a few TeVs [21], the HCA model can be tested in contemporary and future experiments in particle colliders.

Inspired by the now refuted reports on the $750 \mathrm{GeV}$ excess, we initiated a general investigation to determine the possible detection of the HCA in contemporary and future colliders. We followed a standard procedure used most often for phenomenological studies of this kind. (See, e.g., [10-13]. In most of these cases, the suggested new particle couples to additional operators, such as $Y_{\mu \nu} Y^{\mu \nu}$, rather than exclusively to $Y_{\mu \nu} \tilde{Y}^{\mu \nu}$ like the HCA. However, the phenomenological procedures are similar to the one performed for the HCA.) In our investigation we identified the experimental signatures of the HCA, designed a data analysis strategy that maximizes sensitivity to the HCA model, and evaluated how many data are required to convincingly establish, or rule out, the model.

This paper is organized as follows: In Sec. II we present a general setup of the HCA model and discuss theoretical and phenomenological characteristics of the HCA. In Sec. III, we present criteria for detection and an updated analysis for the possible detection of the HCA at the LHC, as well as at the High Luminosity LHC (HL-LHC) and other future colliders. Some final comments are made in Sec. IV.

\section{GENERAL SETUP OF THE HCA MODEL}

In [15], Brustein and Oaknin discuss hypercharge electrodynamics in the unbroken phase of the electroweak plasma coupled to a cosmological pseudoscalar. We, however, treat below a simpler form of the Lagrangian studied by Brustein and Oaknin; we focus on a singlet elementary HCA whose only coupling to SM fields is to hypercharge fields.

Pseudoscalar fields with axionlike coupling appear in several possible extensions of the SM and typically have only perturbative derivative interactions and therefore vanishing potential. They acquire mass through nonperturbative interactions. Nonperturbative effects generate a potential of the form $V(\phi)=V_{0}^{4} V(\phi / f)$, where $V$ is a bounded periodic function characterized by the mass generation scale, $f$, also known as the "Peccei-Quinn" scale. The scale $f$ could be as high as the Planck scale or much lower, even down to the $\mathrm{TeV}$ range.

By coupling the HCA, $X$, to hypercharge electromagnetic fields, $Y_{\mu \nu}$, and considering for simplicity $V(X / f)=$ $\Lambda^{4}(1-\cos X / f)$, we find that the SM Lagrangian is supplemented by

$L=\frac{1}{2}\left(\partial_{\mu} X\right)^{2}-\Lambda^{4}\left(1-\cos \frac{X}{f}\right)-\frac{1}{4 M} \epsilon^{\mu \nu \rho \sigma} X Y_{\mu \nu} Y_{\rho \sigma}$.

Note the following:

(i) The HCA can be displaced from its minimum in the early universe with interesting consequences, but for now we assume that it has reached its global minimum at $X=0$, where its mass is given by $m_{X} \equiv \Lambda^{2} / f \ll M$. The right-hand side (RHS) of the last expression ensures small radiative corrections (see below).

(ii) The mass acquired by the HCA could be as low as a fraction of an $\mathrm{eV}$, or as high as $10^{12} \mathrm{GeV}$. A particularly interesting mass range is the $\mathrm{TeV}$ range, expected to appear if mass generation is associated with supersymmetry breaking and if the HCA plays a role in baryogenesis [15].

(iii) The scales $M$ in the hypercharge sector and $\Lambda, f$ in the mass generation sector are not related. Our model is therefore a two parameter model, and the goal of our analysis is to determine for which domains in $\left(m_{X}, M\right)$ space HCA can be produced and detected in colliders.

The part of the Lagrangian that is of interest for detection of a HCA is the last term in Eq. (1). We have decomposed the interaction term into the ordinary photon and $Z$ boson and obtained the couplings shown in Fig. 1. Next, we calculated partial decay widths, and these are the expressions obtained:

$$
\begin{aligned}
\Gamma_{X \rightarrow \gamma \gamma} & =\frac{1}{64 \pi M^{2}} \cos ^{4} \theta_{W}\left[m_{X}^{3}\right], \\
\Gamma_{X \rightarrow Z_{\gamma}} & =\frac{2}{64 \pi M^{2}} \cos ^{2} \theta_{W} \sin ^{2} \theta_{W}\left[\frac{\left(m_{X}^{2}-m_{Z}^{2}\right)^{3}}{m_{X}^{3}}\right], \\
\Gamma_{X \rightarrow Z Z} & =\frac{1}{64 \pi M^{2}} \sin ^{4} \theta_{W}\left[\left(m_{X}^{2}-4 m_{Z}^{2}\right)^{3 / 2}\right], \\
\Gamma & =\Gamma_{\gamma \gamma}+\Gamma_{Z \gamma}+\Gamma_{Z Z} .
\end{aligned}
$$




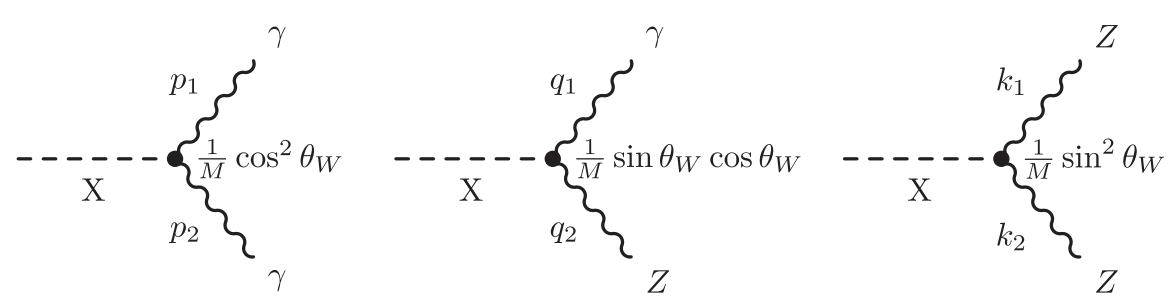

FIG. 1. The HCA couplings to photons and $Z$ bosons.

The branching ratios of a HCA whose mass ranges few TeVs depend weakly on its mass, $m_{X}$, and do not depend on the coupling $1 / M$. Moreover, we found that $\Gamma_{\gamma \gamma} / \Gamma$ dominates throughout the range of parameters researched.

\section{A. Phenomenological methods}

Throughout this study, events were generated in MADGRAPH5_AMC@NLO, a Monte Carlo event generator used most often for simulating particle colliders [22]. Cross sections for production processes of the HCA were evaluated using a simulation code that fits the Brustein and Oaknin proposed theory; the effective operator in Eq. (1) had been implemented ${ }^{1}$ in model Universal FeynRules Output files created with FeynRules [23].

We have conducted several validation tests on MADGRAPH5_AMC@NLO with well known cross sections in perturbation theory, as well as with processes which involve the HCA. All validation tests were successful; numerical simulations reproduced complete perturbative results to $\mathrm{O}(0.1 \%)$.

\section{B. Production mechanisms}

The HCA can be produced at high energy colliders via vector boson fusion (VBF) or via associated production (AP) with another photon or $Z$ boson. Both VBF and AP are depicted in Fig. 2.

The AP channel had been studied by Brustein and Oaknin [21]. Using the AP diagrams shown in Fig. 2, we calculated the total unpolarized cross sections for the two processes $f \bar{f} \rightarrow Z^{*}, \gamma^{*} \rightarrow Z X$ and $f \bar{f} \rightarrow Z^{*}, \gamma^{*} \rightarrow \gamma X$ and obtained

$$
\begin{aligned}
\sigma(f \bar{f} \rightarrow X Z)= & \frac{\alpha}{12 N_{c}} \frac{1}{M^{2}} \sqrt{\hat{s}} E_{z}^{3} \rho^{3} \frac{\sin ^{2} \theta_{w}}{\cos ^{2} \theta_{w}} \\
& \times\left\{\left[\frac{c_{V}}{\hat{s}-m_{z}^{2}}+2 \frac{\cos ^{2} \theta_{w}}{\hat{s}}\left(g_{A} / e\right)\right]^{2}\right. \\
& \left.+\frac{c_{A}^{2}}{\left(\hat{s}-m_{z}^{2}\right)^{2}}\right\},
\end{aligned}
$$

\footnotetext{
${ }^{1}$ Specifically for a HCA of mass $m_{X}=750 \mathrm{GeV}$, and $M=100 \mathrm{TeV}$. The coupling and parameter adjustments required for our analysis are easy to make using MADGRAPH5_AMC@NLO.
}
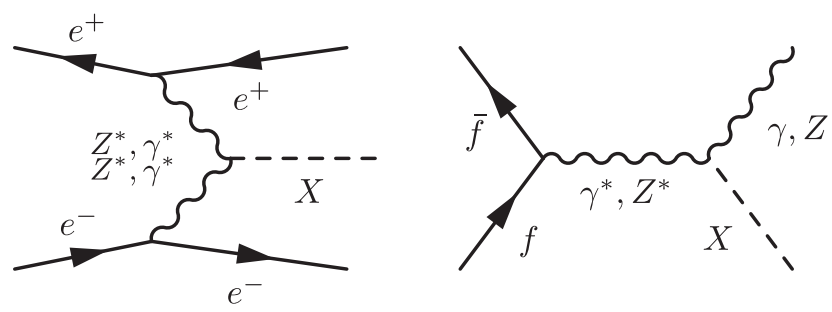

FIG. 2. Production of the HCA via (left) VBF and (right) AP. $Z^{*}, \gamma^{*}$ are virtual bosons. The fermions can be either charged leptons or quarks. At hadron colliders, the VBF-induced HCA production is treated as a double deep-inelastic scattering (see below).

$$
\begin{aligned}
\sigma(f \bar{f} \rightarrow X \gamma)= & \frac{\alpha}{12 N_{c}} \frac{1}{M^{2}} \sqrt{\hat{s}} E_{\gamma}^{3} \\
& \times\left\{\left[\frac{c_{V}}{\hat{s}-m_{z}^{2}}+2 \frac{\cos ^{2} \theta_{w}}{\hat{s}}\left(g_{A} / e\right)\right]^{2}\right. \\
& \left.+\frac{c_{A}^{2}}{\left(\hat{s}-m_{z}^{2}\right)^{2}}\right\},
\end{aligned}
$$

where $E_{Z}=\frac{m_{Z}^{2}-m_{X}^{2}+\hat{s}}{2 \sqrt{\hat{s}}}, \rho=\sqrt{1-\frac{m_{Z}^{2}}{E_{Z}^{2}}}, E_{\gamma}=\frac{\hat{s}-m_{X}^{2}}{2 \sqrt{\hat{s}}}, N_{c}$ is the number of colors of the fermion which takes into account averaging over initial colors, and $\sqrt{\hat{s}}$ denotes the parton center of mass (CM) energy. In deriving Eqs. (3) and (4) we assumed that the fermions are effectively massless, $m_{f} \ll \sqrt{\hat{s}}$. The parameters appearing in Eqs. (3) and (4) are given in Table I: $c_{V}$ and $c_{A}$ are the vector and axial coupling of the fermion to the $Z$ boson.

Notice that for small $\hat{s}$, there are kinematical thresholds for both processes, $\hat{s}>m_{X}^{2}$ and $\hat{s}>\left(m_{X}+m_{Z}\right)^{2}$, to allow AP with a photon and with a $Z$ boson, respectively. Additionally, since $E_{\gamma}, E_{Z} \sim \sqrt{\hat{s}}$ for large $\hat{s}$, both cross

TABLE I. Parameter values for fermions. $\alpha$ denotes the fine structure constant.

\begin{tabular}{lccc}
\hline \hline$f$ & $g_{A}$ & $c_{V}$ & $c_{A}$ \\
\hline$e^{-}, \mu^{-}, \tau^{-}$ & $e=\sqrt{4 \pi \alpha}$ & $-\frac{1}{2}+2 \sin ^{2} \theta_{w}$ & $-\frac{1}{2}$ \\
$u, c, t$ & $-\frac{2}{3} \sqrt{4 \pi \alpha}$ & $\frac{1}{2}-\frac{4}{3} \sin ^{2} \theta_{w}$ & $\frac{1}{2}$ \\
$d, s, b$ & $\frac{1}{3} \sqrt{4 \pi \alpha}$ & $-\frac{1}{2}+\frac{2}{3} \sin ^{2} \theta_{w}$ & $-\frac{1}{2}$ \\
\hline \hline
\end{tabular}



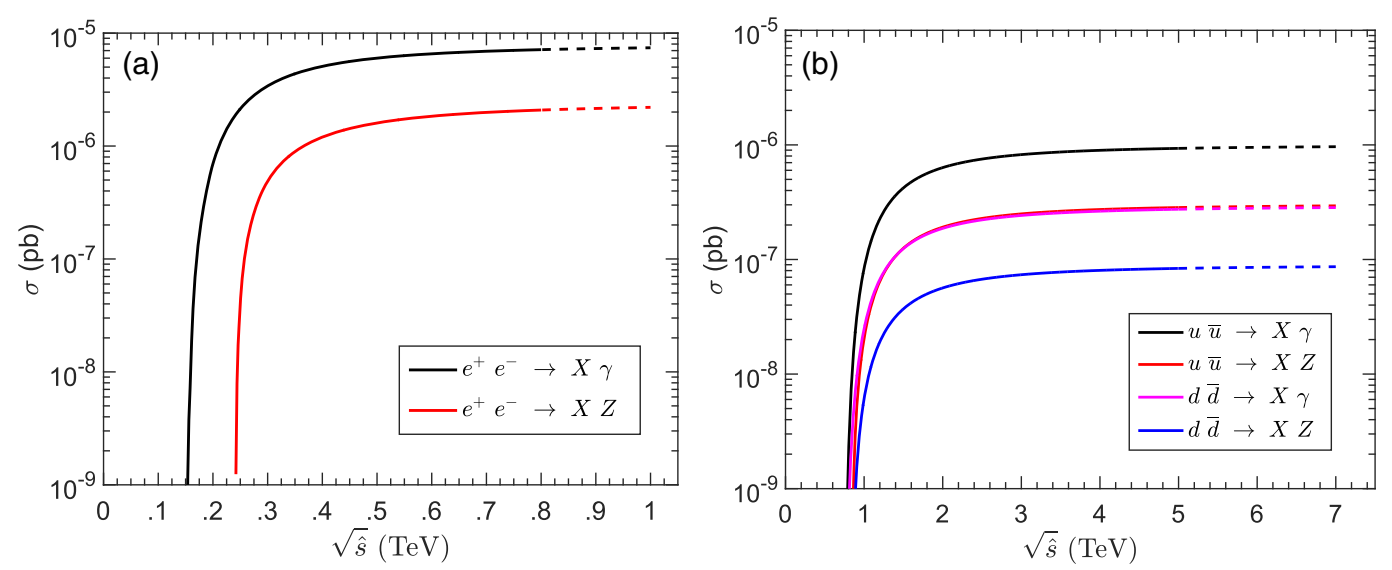

FIG. 3. Cross sections for $\mathrm{AP}$ processes as a function of the partons $\mathrm{CM}$ energy at (a) $e^{+} e^{-}$colliders, for $m_{X}=150 \mathrm{GeV}$, $M=100 \mathrm{TeV}$, and (b) hadron colliders, for $m_{X}=750 \mathrm{GeV}, M=100 \mathrm{TeV}$.

sections approach asymptotically a constant [apart from a logarithmic dependence of $\alpha(\sqrt{\hat{s}})]$ independent of the mass $m_{X}$, such that for large $\hat{s}, \frac{\sigma(f \bar{f} \rightarrow Z X)}{\sigma(f \bar{f} \rightarrow \gamma X)} \simeq \tan ^{2} \theta_{W} \simeq 0.3$. The rise toward the asymptotic value is governed by the ratios $m_{X}^{2} / \hat{s}$ and $m_{Z}^{2} / \hat{s}$.

In order to evaluate cross sections at hadron colliders such as the LHC, we used the parton model. For both AP processes, the only possible contributions are from the six same-flavor quark-antiquark partonic collisions, whose cross sections are given in Eqs. (3) and (4).

The AP cross sections calculated using perturbation theory, Eqs. (3) and (4), were checked against MADGRAPH5 AMC@NLO results. The software uses constantly updated parton distribution functions of protons in order to simulate $p p$ collisions. In Fig. 3 we present the AP cross sections as a function of the parton CM energy at (a) $e^{+} e^{-}$colliders, for $m_{X}=150 \mathrm{GeV}$ and $M=100 \mathrm{TeV}$, and at (b) hadron colliders for $m_{X}=750 \mathrm{GeV}$ and $M=100 \mathrm{TeV}$.

The VBF mechanism and possible detection of HCAs at the ATLAS detector were studied by Elfgren [24]. An analytic expression of cross sections for VBF processes unfortunately is not very illuminating as it depends on the vector-boson distribution functions in the colliding fermions. Nonetheless, in order to get a rough estimate of the processes' cross sections we use the Weizsacker-Williams approximation [25], also known as the equivalent photon approximation (EPA). The EPA seems to give a reasonable order of magnitude estimate of the cross section. The EPA estimate becomes better for $\mathrm{CM}$ energies that are much higher than $m_{X}^{2}$.
The Weizsacker-Williams photon spectrum, $f_{f / \gamma}(x)$, is the photon distribution with momentum fraction $x$ in a chargedparticle beam of energy $E$. For an electron, or a light quark of energy $E$, the probability of finding a collinear photon of energy $x E$ is given by

$$
f_{f / \gamma}(x)=\frac{g_{A}^{2}}{8 \pi^{2}} \frac{x+(1-x)^{2}}{x} \ln \left(\frac{t_{\max }}{t_{\min }}\right) .
$$

Here $t_{\max }$ and $t_{\min }$ are the characteristic maximum and minimum photon momentum transfers and $g_{A}$ is given in Table I. For the process under consideration, the production of heavy HCA of mass $m_{X}$, we take these to be $t_{\min }=1 \mathrm{GeV}^{2}$ and $t_{\max }=\hat{s}$, with $\hat{s}$ being the partonic $\mathrm{CM}$ energy. There is some flexibility in the choice of $t_{\max }$. However, the results are not very sensitive to this parameter within the limits of the Weizsacker-Williams approximation [26]. The particular choice of the minimum momentum transfer, $t_{\min }$, guarantees that the photons are obtained from the deep inelastic scattering of protons and the quark-parton model is valid.

One could generalize EPA to what is known as the effective vector-boson approximation (EVBA) [27,28] for processes with weak bosons in place of photons [26]. That, however, cannot be done analytically and is beyond the scope of this study. In addition, the production of a HCA whose mass is within the range researched of few $\mathrm{TeVs}$ is dominated by the exchange of two photons. We therefore content ourselves with HCA production via two photons only and obtain an expression for the parton-level cross section

$$
\begin{aligned}
\sigma^{\text {inel }}(\hat{s}) & \approx \int d x f_{f / \gamma}(x) \int d y f_{f / \gamma}(y) \sigma(\gamma \gamma \rightarrow X, x y \hat{s}) \\
& =\int_{0}^{1} d x f_{f / \gamma}(x) \int_{0}^{1} d y f_{f / \gamma}(y) \frac{\left\langle|\mathcal{M}|^{2}\right\rangle}{2(2 x E)\left(2 y E^{\prime}\right)} 2 \pi \delta\left(\left(k+k^{\prime}\right)^{2}+m_{X}^{2}\right) .
\end{aligned}
$$


Here $\mathcal{M}$ denotes the invariant amplitude for production of the HCA in a collision of two photons with momenta $k, k^{\prime}$, in a charged-particle beam of energies $E, E^{\prime}$, respectively.
Integrating over $y$ using the $\delta$-function, averaging over initial colors, and expressing $\left\langle|\overline{\mathcal{M}}|^{2}\right\rangle$ in terms of $\Gamma(X \rightarrow \gamma \gamma)$, we find

$$
\begin{aligned}
\sigma^{\text {inel }}(\hat{s}) & =\frac{16 \pi^{2} \Gamma(X \rightarrow \gamma \gamma)}{m_{X} \hat{s} N_{c}} \int_{m_{X}^{2} / \hat{s}}^{1} \frac{d x}{x} f_{f / \gamma}(x) f_{f / \gamma}\left(m_{X}^{2} / x \hat{s}\right) \\
& \equiv \frac{16 \pi^{2} \Gamma(X \rightarrow \gamma \gamma)}{m_{X} \hat{s} N_{c}} \frac{d L_{f f / \gamma \gamma}}{d\left(m_{X}^{2} / \hat{s}\right)}
\end{aligned}
$$

An analytic expression can be obtained for the differential luminosity. Thus, by substituting $\Gamma(X \rightarrow \gamma \gamma)$ [Eq. (2)], we obtain

$$
\sigma^{\text {inel }}(\hat{s}) \approx \frac{\pi \alpha^{2}}{256 \hat{s} N_{c}}\left(\frac{g_{A}}{e}\right)^{4} \frac{m_{X}^{2}}{M^{2}} \cos ^{4} \theta_{W}\left[\left(4-\frac{6 \hat{s}}{m_{X}^{2}}+\frac{2 m_{X}^{2}}{\hat{s}}\right)-\left(4+\frac{4 \hat{s}}{m_{X}^{2}}+\frac{m_{X}^{2}}{\hat{s}}\right) \ln \frac{m_{X}^{2}}{\hat{s}}\right]\left(\ln \frac{t_{\mathrm{max}}}{t_{\min }}\right)^{2} .
$$

The approximation is only valid when the transverse momentum of the fermions is virtually zero and when they are ultrarelativistic. This should be a fairly good approximation, as the fermions are either high energy electrons in $e^{+} e^{-}$colliders or the quarks inside a proton which have very little transverse momentum and a relativistic velocity.

In Fig. 4 we present cross sections for production of the HCA via VBF as a function of $\hat{s}$ at hadronic and $e^{+} e^{-}$colliders. We see that EPA can reproduce the order of magnitude of the complete perturbative results. The approximation is valid for $\mathrm{CM}$ energies much larger than $\hat{s} \sim m_{X}^{2}$. For very large CM energy $\left(m_{Z}^{2} \ll \hat{s}\right)$ one has to consider contributions from production via $Z \gamma$ and $Z Z$.

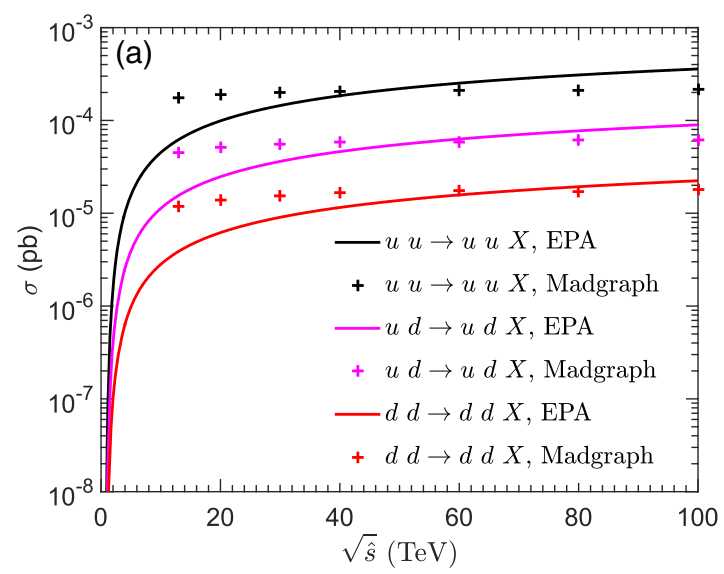

According to Figs. 5 and 6, the most probable production mechanism of the HCA at hadron colliders is the VBF, whereas at $e^{+} e^{-}$machines, the production of the HCA in association with another photon is favorable.

\section{Decay channels}

Assuming that $m_{X} \geq 2 m_{Z}$, all vertices in the HCA model (Fig. 1) are kinematically allowed. The HCA can therefore decay via all three channels. Equations (2) suggest that widths in the $\mathrm{GeV}$ range can be expected for $m_{X} \lesssim M \sim \mathrm{TeV}$. If there is a larger hierarchy between the scales, the width can be much smaller.

Theoretically, large widths can be accommodated [29]. That being said, a narrower width gives a "healthier"
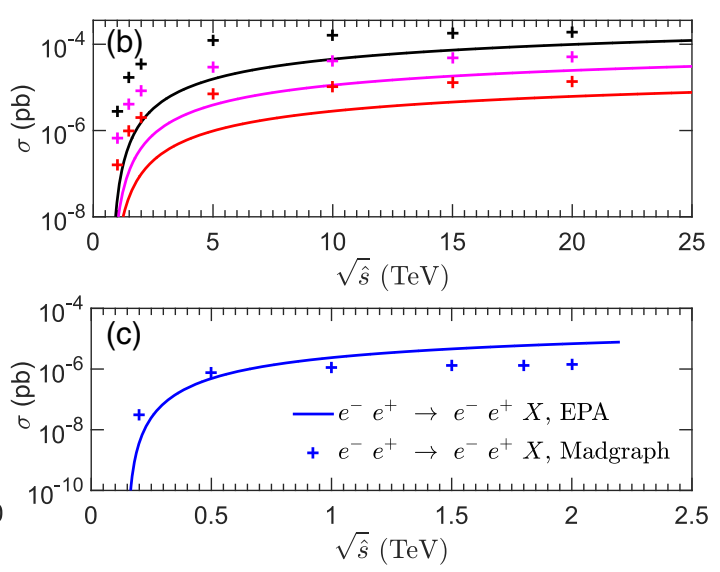

FIG. 4. Parton-level cross sections for VBF processes at (a),(b) hadronic colliders with $m_{X}=750 \mathrm{GeV}, M=10 \mathrm{TeV}$ and (c) $e^{+} e^{-}$ colliders with $m_{X}=150 \mathrm{GeV}, M=100 \mathrm{TeV}$ vs parton CM energy. For clarity, only the leading VBF processes at $p p$ colliders are presented. The solid lines are valid to all light quarks of the type specified in the legend. EPA estimates correctly the order of magnitude of the perturbative results only for CM energies much larger than $m_{X}^{2}$. For $m_{Z}^{2} \ll \hat{s}$ one has to consider contributions from production processes via $Z \gamma$ and $Z Z$. 

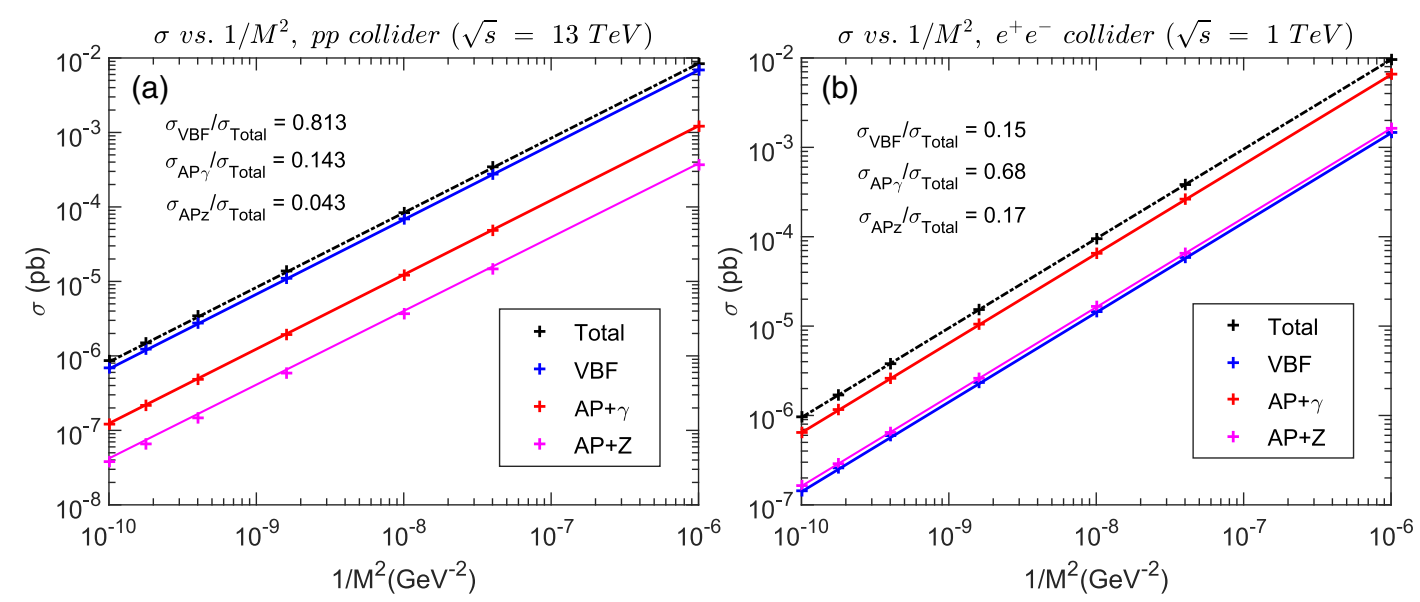

FIG. 5. HCA production $\left(m_{X}=750 \mathrm{GeV}\right)$ at (a) the LHC and (b) a next generation $e^{+} e^{-}$collider. All lines are fitted curves. VBF is the most probable production process at hadron colliders, whereas at $e^{+} e^{-}$colliders, the production of the HCA in association with a photon is favorable.
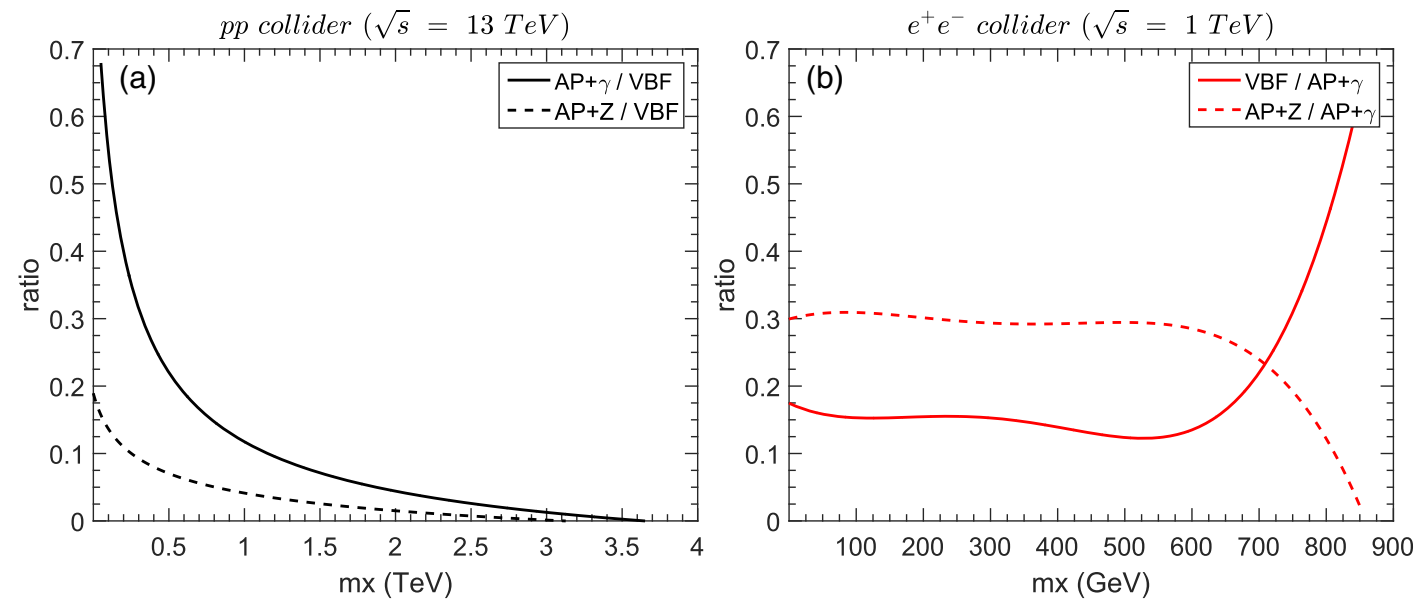

FIG. 6. (a) Ratios of cross sections for AP of HCA with a photon and with a $Z$ boson, to cross section for production via VBF at the LHC. (b) Ratios of cross sections for AP of HCA with a $Z$ boson and production via VBF, to cross section for AP with a photon at a next generation $e^{+} e^{-}$collider. In both cases $M=10 \mathrm{TeV}$.

model; the interaction term is not renormalizable, and therefore our model should be considered as an effective field theory, with a cutoff. Loop corrections could thus present a problem, or even invalidate our analysis, if they are as large as the tree level ones. This concern is certainly relevant in the case $m_{X} \sim M$, as the relative magnitude of a loop with external gauge bosons and an internal HCA, compared to the same process at tree level, is approximately of order $\frac{1}{16 \pi^{2}} \frac{m_{X}^{2}}{M^{2}}$, assuming that the HCA is the heaviest particle running in the loop [21]. However, if we manage to stay within a realm where $m_{X}<M$, expected radiative corrections are small. Moreover, we recall that the HCA potential is generated by nonperturbative effects. As such, naive dimensional analysis does not necessarily hold. We will not attempt here a more detailed treatment of radiative corrections.
According to Figs. 5 and 6, the dominant production mechanism depends on the collider type. Therefore, for the sake of generality, we characterize the HCA using its decay products only. Having said that, decay products of a VBFinduced HCA production are expected to be emitted backto-back, generally with high $p_{T}$. In addition, the AP key signature is, as well, a very distinctive event with a three photon final state. The SM background for the three photon final state at $e^{+} e^{-}$colliders is mainly a pure QED process, whose differential cross section is strongly peaked along the forward and backward directions [30,31], whereas the three photons coming from near resonance AP and decay of the HCA were found to be isotropically distributed. These properties can be used to distinguish the HCA signal from background events, and thus serve as very powerful checks for verifying discovery. 
TABLE II. Decay modes of the HCA, branching ratios, and detectable final states.

\begin{tabular}{lccc}
\hline \hline $\begin{array}{l}\text { Decay } \\
\text { modes }\end{array}$ & $\begin{array}{c}\text { Branching } \\
\text { ratio }\end{array}$ & $\begin{array}{c}\text { Detectable } \\
\text { final state }\end{array}$ & $\begin{array}{c}\text { Branching } \\
\text { ratio }\end{array}$ \\
\hline$\gamma \gamma$ & $59 \%$ & $\gamma \gamma$ & $100 \%$ \\
$\gamma Z$ & $35 \%$ & $\gamma l \bar{l}$ & $6.7 \%$ \\
$Z Z$ & & $\gamma q q$ & $70 \%$ \\
& $6 \%$ & $l \bar{l} l \bar{l}$ & $0.45 \%$ \\
& & $l \bar{l} q q$ & $9.4 \%$ \\
& & $q q q q$ & $49 \%$ \\
\hline \hline
\end{tabular}

To characterize the HCA final decay products at particle detectors, we examine the $Z$ boson decay products[32]. The $Z$ decays to two leptons of each flavor $3.3 \%$ of the time, to two quarks $70 \%$ of the time, and into neutrinos the rest of the time. The $\tau$ is more difficult to detect at the LHC and is not considered here, leaving the electron and the muon for a total of $6.7 \%$ of the detectable lepton decays. We thus obtain the HCA final decay products with their respective ratios that are shown in Table II.

The final decay products of the HCA appear with fixed ratios and can therefore serve as a powerful diagnostic for verifying discovery of the HCA; if several different final states are detected in the predicted ratios, the new particle could be identified as the HCA.

Since $X \rightarrow Z+Z$ is rare, and $X \rightarrow \gamma+$ jet + jet is very polluted by the QCD background at hadron colliders, the most promising final states for detection are

$$
\begin{aligned}
& X \rightarrow \gamma+\gamma, \\
& X \rightarrow \gamma+Z \rightarrow \gamma+l+\bar{l} .
\end{aligned}
$$

\section{DETECTION AT PRESENT AND FUTURE COLLIDERS}

Finally, we present the estimated number of produced HCAs in colliders, $N_{\mathrm{ev}}$. Equations (3), (4), and (8), along with the expression $N_{\text {ev }}=\mathcal{L}_{\text {int }} \cdot \sigma$, where $\mathcal{L}$ is the integrated luminosity of the experiment, relate $N_{\mathrm{ev}}$ and the two parameters of the model: the HCA mass and the coupling.

If we fix a number of events as the minimal number $N_{\text {min }}$ required for detection of the HCA, we obtain an "accessible detection range" in $\left(m_{X}, M\right)$ space, limited by the curve

$$
\frac{M}{\mathrm{TeV}}=\sqrt{\frac{\mathcal{L}_{\text {int }}}{N_{\text {min }}}} \sqrt{\sigma\left(M=1 \mathrm{TeV}, s, m_{x}\right)} .
$$

In Fig. 7, this curve is shown for $N_{\min }=10$ for Run II of the LHC, along with an exclusion region obtained from previous experiments (see below). The reach of future colliders, amongst which are future runs of the LHC, HL-LHC, and a hadronic future circular collider, is shown
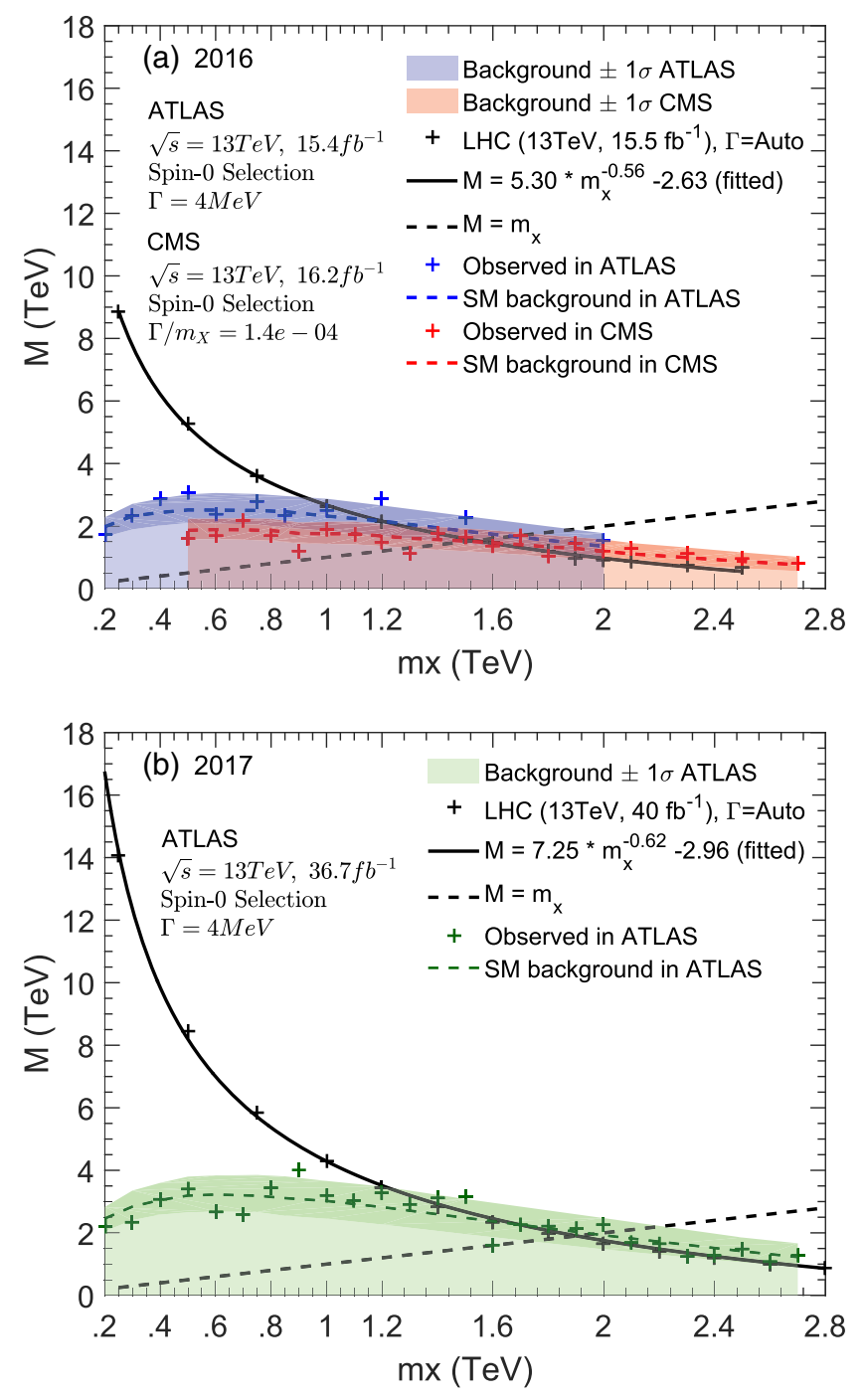

FIG. 7. Expected number of HCAs produced in Run II of the LHC; $\sqrt{s}=13 \mathrm{TeV}$ (a) $\mathcal{L}_{\text {int }}=15.5 \mathrm{fb}^{-1}$, collected with the LHC detectors during 2015 and 2016, and (b) $\mathcal{L}_{\text {int }}=40 \mathrm{fb}^{-1}$, collected with the ATLAS detector by the end of 2017. The shaded regions are excluded for different decay widths. Note that different cuts were used in the event selection procedures of ATLAS and CMS analyses ([8,33, Secs. 4-7] and [7], respectively). The solid lines correspond to ten expected events.

in Fig. 8. For the graphs in Figs. 7 and 8, we have chosen some generic parameters characterizing the various machines, summarized in Table III.

In the region below the curves more than ten events are expected with luminosities given in legends, while in the region above the curves fewer than ten events are expected. Since graphs scale with parameters as in Eq. (10), it is simple to adapt them to different parameters characterizing the various machines.

Our choice of $N_{\min }=10$ events is motivated by the expectation that the SM background for the processes is small. The actual reach of future colliders for detection of the HCA will be determined by the background. 


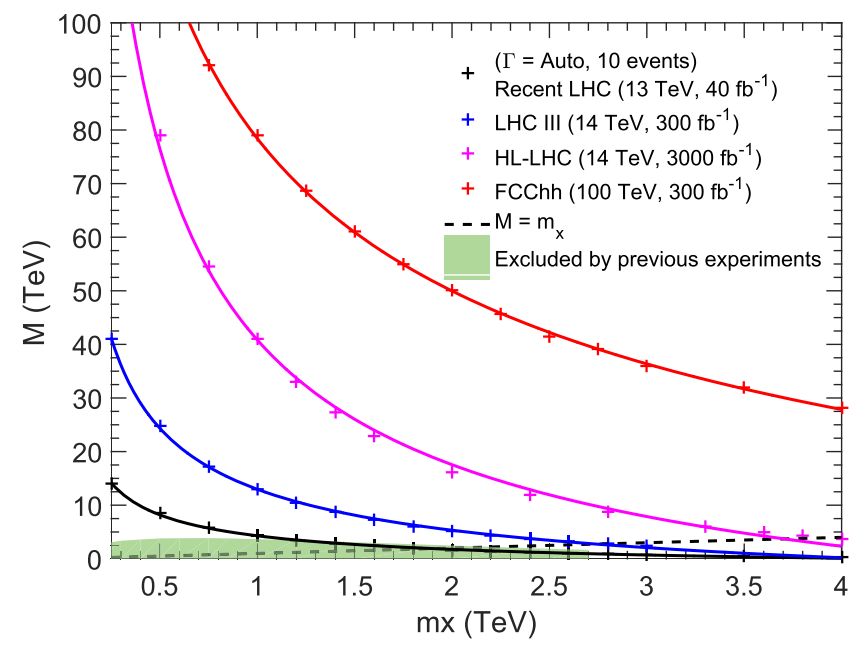

FIG. 8. Expected number of HCAs produced in future runs of the LHC, HL-LHC, and about one year of a hadronic future circular collider. The solid lines are fitted curves which correspond to ten expected events; below (above) the curve more (less) than ten events are expected, with integrated luminosity given in the legend. The shaded region is experimentally excluded.

The HCA had not been detected so far. Nonetheless, we can use existing experimental data from recent particle experiments to rule out significant regions in the model's parameter space. In order to do so, we use the analyses in [7,33] and the more recent one in [8]. These analyses summarize the results of recent searches for high-mass diphoton resonances with different widths, specifically spin-0 and spin-2 resonances with an invariant mass between hundereds of $\mathrm{GeVs}$ to few TeVs in $p p$ collisions at a CM energy of $13 \mathrm{TeV}$. The data samples correspond to an integrated luminosity of $15.4 \mathrm{fb}^{-1}$ collected with the ATLAS detector and $16.2 \mathrm{fb}^{-1}$ collected with CMS during 2015 and 2016, and $36.7 \mathrm{fb}^{-1}$ collected with the ATLAS detector by the end of 2017 ([7,8,33], respectively). No

TABLE III. Table of parameter values for high-energy colliders [32]. The parameters expected at the LHC experiments for the 2018 run and the design values for the High-Luminosity upgrade (HL-LHC) are also shown.

\begin{tabular}{lcccc}
\hline \hline Name & Type & $\mathcal{L}_{\text {int }}\left(\mathrm{fb}^{-1}\right)$ & $\sqrt{s}(\mathrm{TeV})$ & Years of operation \\
\hline LHC I & \multicolumn{5}{c}{23.3 at $8 \mathrm{TeV}$} & $7-8$ & $2010-2013$ \\
& 6.1 at $7 \mathrm{TeV}$ & & \\
LHC II & $p p$ & 80 & 13 & $2015-$ now \\
& & $120-150^{\mathrm{a}}$ & 13 & $2015-2018$ \\
LHC III & \multicolumn{5}{c}{$300^{\mathrm{a}}$} & $14^{\mathrm{a}}$ & $2020-2023$ \\
HL-LHC & $p p$ & $250 / \mathrm{y}^{\mathrm{a}}$ & $14^{\mathrm{a}}$ & $2026-2038 ?$ \\
ILC & $e^{+} e^{-}$ & $1.5 \times 10^{-5} / \mathrm{s}^{\mathrm{a}}$ & $0.5-1^{\mathrm{a}}$ & TBD \\
FCC-hh & $p p$ & $0.2-1 \mathrm{M} / \mathrm{y}^{\mathrm{a}}$ & $100^{\mathrm{a}}$ & $? ? ?$ \\
\hline \hline
\end{tabular}

\footnotetext{
${ }^{a}$ Tentative design parameters of selected future high-energy
} colliders. significant excess had been observed relative to the SM expectation.

Since HCAs within the "accessible detection range" in $\left(m_{X}, M\right)$ space, or $\left(m_{X} \sim 1 \mathrm{TeV}, M \sim 10 \mathrm{TeV}\right)$ according to Fig. 8, have narrower decay width of few MeVs, we further examine the graphs which correspond to limits on the signal cross section times the branching ratio to two photons for a spin-0 particle as a function of the assumed signal mass and for narrow-width resonance, as in Fig. 7(a) of [33], Fig. 4(upper) of [7], and Fig. 4(a) of [8]. First, we calculated the total cross section per mass value by dividing the aforementioned plots with the branching ratio to two photons in the HCA model. Then, using MADGRAPH5_AMC@NLO, we calculated $M$ values.

In their study, Brustein and Oaknin analyzed data from Tevatron and LEP II which set upper bounds on the cross sections $\sigma\left(e^{+} e^{-} \rightarrow X Z\right)$, of the order of $0.1 \mathrm{pb}$, for $m_{X}<$ $90 \mathrm{GeV}$ and as assuming that $X \rightarrow \gamma \gamma$ [21]. The updated analysis we performed, summarized in Fig. 7(b), clearly enlarges the exclusion region obtained by Brustein and Oaknin, and thus provides stricter constraints on regions in $\left(m_{X}, M\right)$ space in which the HCA can be produced and detected at the LHC. That being said, a specific analysis with particular details of the HCA model, should be carried out in order to determine the exact exclusion region.

The main results of our analysis concern future colliders and are summarized in Fig. 8. First, we conclude that for the range of masses $m_{X} \lesssim 1 \mathrm{TeV}$, the HCA could be detected even for the $80 \mathrm{fb}^{-1}$ accumulated luminosity available today at a CM energy of $13 \mathrm{TeV}$. Such a large accessible detection region calls, in our opinion, for further experimental attention.

In addition, our results indicate that the accessible detection region increases dramatically as the luminosity and the CM energy increase. Quantitatively one can say that since $N_{\text {sig }} / \sqrt{N_{\text {SM }}} \propto \sqrt{\mathcal{L}_{\text {int }}}$, the HL-LHC is expected to increase the accessible detection range by a factor of $\sqrt{10}$ beyond the accessible region at the LHC by the end of its operational period. Moreover, though there are not any near-future practical intentions of building higher than the LHC energy colliders, we have obtained quite an interesting result that while a luminosity increase provides better reach in coupling $M$, higher collision energy was found able to significantly increase the reach in mass, $m_{X}$, as well.

Before concluding our analysis, it is important to note that though a clear signal for the $X \rightarrow \gamma \gamma$ could support a discovery and verify the branching ratios, it is not enough for discovery. If we want to be certain that it is really the HCA that is detected, the branching ratios of $X \rightarrow \gamma \gamma$ and $X \rightarrow \gamma Z \rightarrow \gamma l \bar{l}$, the second most promising signature, have to be compared. The ratio $\Gamma_{\gamma \gamma} / \Gamma_{\gamma l l}$ should be 25.1 for the HCA. If all three decay channels are discovered, then it is a strong indication that we have discovered a (pseudo)scalar with the right coupling to hypercharge. At that stage, the mass scale $M$ can be used to determine theoretically 
whether the pseudoscalar could amplify the hypermagnetic fields or not [16].

\section{CONCLUSIONS}

Our study focuses on the HCA model, a model that could potentially serve as a viable baryogenesis mechanism. The model has great testability potential by contemporary and future experiments. Our general approach has been illustrated in detail, for what is probably the minimal extension of the SM in this context: the addition of a single (pseudo) scalar field that specifically couples to weak hypercharge.

The hypercharge photon is a linear combination of the ordinary photon and $Z$ boson; hence the hypothetical particle could be produced, in hadronic or leptonic colliders, through $\mathrm{VBF}$, or in association with another photon or $Z$ boson. Figures 5 and 6 show that the VBF process is the most promising production process of the HCA at hadron colliders, whereas at $e^{+} e^{-}$colliders, the production of the HCA in association with another photon is the most probable process.

The produced HCA decays into two neutral gauge bosons, $\gamma \gamma, \gamma Z$, or $Z Z$. The experimental signatures of these processes are, then, two or three neutral gauge bosons produced in well defined ratios. We concluded that there are practically two processes that can be used for detection of the HCA; $X \rightarrow \gamma \gamma$ and $X \rightarrow \gamma Z \rightarrow \gamma l \bar{l}$. The former signal is expected to have the higher signal-to-background ratio. Moreover, we argued that the almost isotropic angular distribution of momenta of the outgoing bosons could help in separating it from the QED background at $e^{+} e^{-}$ colliders.

Our analysis updates a similar analysis performed by Brustein and Oaknin two decades ago, to contemporary and future colliders. It suggests that the accessible HCA parameter region for the detection of the $\mathrm{HCA}$ at future colliders increases dramatically as the luminosity and CM energy increase; higher effective luminosity increases the reach in coupling, while higher energy increases the reach in mass. In addition, our analysis significantly broadens the exclusion region obtained by Brustein and Oaknin, and thus provides stricter constraints on domains in the model's parameter space in which the HCA can be produced and detected at colliders.

An additional important implication of our results is that there are parameter regions where the potential of detecting the HCA has not yet been exhausted. We find that even for the $80 \mathrm{fb}^{-1}$ accumulated luminosity available today at a $\mathrm{CM}$ energy of $13 \mathrm{TeV}$, the region of parameter space of relatively small mass is still not excluded. This calls, in our opinion, for further experimental attention. One could additionally use recent reports by ATLAS and CMS Collaborations $[34,35]$ in order to study the second most "detectable" signature, $X \rightarrow \gamma Z \rightarrow \gamma l \bar{l}$, and use it to help in verifying branching ratios in particular and a discovery in general.

Due to lack of experimental data, the analysis presented here unfortunately does not cover certain necessary aspects of a meaningful search, which may easily be integrated and complement our research, such as systematic uncertainties elimination, background estimations at future colliders, and more. Hopefully, our study will inspire particle experimenters, or a future study in general, to further examine these and other aspects of the HCA model in more detail.

\section{ACKNOWLEDGMENTS}

We would like to thank Liron Barak, Yevgeny Kats, and David Oaknin for many helpful discussions. The research was supported by the Israel Science Foundation Grant No. 1294/16.
[1] L. Canetti, M. Drewes, and M. Shaposhnikov, Matter and antimatter in the Universe, New J. Phys. 14, 095012 (2012).

[2] M. M. Anber and E. Sabancilar, Hypermagnetic fields and baryon asymmetry from pseudoscalar inflation, Phys. Rev. D 92, 101501 (2015).

[3] K. Kamada and A. J. Long, Baryogenesis from decaying magnetic helicity, Phys. Rev. D 94, 063501 (2016).

[4] K. Kamada and A. J. Long, Evolution of the baryon asymmetry through the electroweak crossover in the presence of a helical magnetic field, Phys. Rev. D 94, 123509 (2016).

[5] M. S. Turner and L. M. Widrow, Inflation produced, large scale magnetic fields, Phys. Rev. D 37, 2743 (1988).

[6] E. I. Guendelman and D. A. Owen, Axion driven baryogenesis, Phys. Lett. B 276, 108 (1992).
[7] V. Khachatryan et al. (CMS Collaboration), Search for highmass diphoton resonances in proton-proton collisions at $13 \mathrm{TeV}$ and combination with $8 \mathrm{TeV}$ search, Phys. Lett. B 767, 147 (2017).

[8] M. Aaboud et al. (ATLAS Collaboration), Search for new phenomena in high-mass diphoton final states using $37 \mathrm{fb}^{-1}$ of proton-proton collisions collected at $\sqrt{s}=13 \mathrm{TeV}$ with the ATLAS detector, Phys. Lett. B 775, 105 (2017).

[9] R. Garisto, Editorial: Theorists React to the CERN $750 \mathrm{GeV}$ Diphoton Data, Phys. Rev. Lett. 116, 150001 (2016).

[10] C. Cski, J. Hubisz, and J. Terning, Minimal model of a diphoton resonance: Production without gluon couplings, Phys. Rev. D 93, 035002 (2016); C. Cski, J. Hubisz, S. Lombardo, and J. Terning, Gluon versus photon production 
of a $750 \mathrm{GeV}$ diphoton resonance, Phys. Rev. D 93, 095020 (2016).

[11] S. Fichet, G. von Gersdorff, and C. Royon, Scattering light by light at $750 \mathrm{GeV}$ at the LHC, Phys. Rev. D 93, 075031 (2016); Measuring the Diphoton Coupling of a $750 \mathrm{GeV}$ Resonance, Phys. Rev. Lett. 116, 231801 (2016).

[12] W. Altmannshofer, J. Galloway, S. Gori, A. L. Kagan, A. Martin, and J. Zupan, $750 \mathrm{GeV}$ diphoton excess, Phys. Rev. D 93, 095015 (2016).

[13] L. A. Harland-Lang, V. A. Khoze, and M. G. Ryskin, The production of a diphoton resonance via photon-photon fusion, J. High Energy Phys. 03 (2016) 182.

[14] R. Brustein and D. H. Oaknin, Electroweak Baryogenesis Induced by a Scalar Field, Phys. Rev. Lett. 82, 2628 (1999).

[15] R. Brustein and D. H. Oaknin, Amplification of hypercharge electromagnetic fields by a cosmological pseudoscalar, Phys. Rev. D 60, 023508 (1999).

[16] R. Brustein and D. H. Oaknin, Baryon number asymmetry induced by coherent motions of a cosmological axion like pseudoscalar, in COSMO-99: Proceedings of the Third International Workshop on Particle Physics and the Early Universe (World Scientific, Singapore, 2000).

[17] D. Jimnez, K. Kamada, K. Schmitz, and X. J. Xu, Baryon asymmetry and gravitational waves from pseudoscalar inflation, J. Cosmol. Astropart. Phys. 12 (2017) 011.

[18] A. Kusenko, K. Schmitz, and T. T. Yanagida, Leptogenesis via Axion Oscillations after Inflation, Phys. Rev. Lett. 115, 011302 (2015).

[19] J.E. Kim, Light pseudoscalars, particle physics and cosmology, Phys. Rep. 150, 1 (1987).

[20] R. Brustein and D. H. Oaknin, Candidates for hypercharge axion in extensions of the standard model, Phys. Rev. D 63, 055002 (2001).

[21] R. Brustein and D. H. Oaknin, Signatures of hypercharge axions in colliders, Phys. Rev. D 62, 015001 (2000).

[22] J. Alwall, R. Frederix, S. Frixione, V. Hirschi, F. Maltoni, O. Mattelaer, H.-S. Shao, T. Stelzer, P. Torrielli, and M. Zaro, The automated computation of tree-level and next-toleading order differential cross sections, and their matching to parton shower simulations, J. High Energy Phys. 07 (2014) 079; J. Alwall, M. Herquet, F. Maltoni, O. Mattelaer, and T. Stelzer, MadGraph 5: Going beyond, J. High Energy Phys. 06 (2011) 128.

[23] A. Alloul, N. D. Christensen, C. Degrande, C. Duhr, and B. Fuks, FeynRules 2.0-A complete toolbox for tree-level phenomenology, Comput. Phys. Commun. 185, 2250 (2014).

[24] E. Elfgren, Detection of a hypercharge axion in ATLAS: A Monte Carlo simulation of a pseudoscalar particle (hypercharge axion) with electroweak interactions for the ATLAS detector in the Large Hadron Collider at CERN, in Fundamental Interactions (World Scientific, Singapore, 2002), pp. 185-191.

[25] C. F. von Weizsacker, Radiation emitted in collisions of very fast electrons, Z. Phys. 88, 612 (1934); E. J. Williams, Nature of the high-energy particles of penetrating radiation and status of ionization and radiation formulae, Phys. Rev. 45, 729 (1934).

[26] G. Bhattacharya, P. Kalyniak, and K. A. Peterson, Photon and $Z$ induced heavy charged lepton pair production at a hadron supercollider, Phys. Rev. D 53, 2371 (1996).

[27] S. Dawson, The effective $W$ approximation, Nucl. Phys. B249, 42 (1985).

[28] G. L. Kane, W. W. Repko, and W. B. Rolnick, The effective $W^{ \pm}, Z^{0}$ approximation for high-energy collisions, Phys. Lett. 148B, 367 (1984).

[29] I. Ben-Dayan and R. Brustein, Hypercharge axion and the diphoton $750 \mathrm{GeV}$ resonance, arXiv:1601.07564.

[30] A. Devoto, S. Di Chiara, and W. W. Repko, Noncommutative QED corrections to $e^{+} e^{-} \rightarrow \gamma \gamma \gamma$ at linear collider energies, Phys. Rev. D 72, 056006 (2005).

[31] F. A. Berends and R. Kleiss, Distributions for electronpositron annihilation into two and three photons, Nucl. Phys. B186, 22 (1981).

[32] C. Patrignani et al. (Particle Data Group Collaboration), Review of particle physics, Chin. Phys. C 40, 100001 (2016).

[33] M. Aaboud et al. (ATLAS Collaboration), Search for scalar diphoton resonances with $15.4 \mathrm{fb}^{-1}$ of data collected at $\sqrt{s}=13 \mathrm{TeV}$ in 2015 and 2016 with the ATLAS detector, Report No. ATLAS-CONF-2016-059.

[34] M. Aaboud et al. (ATLAS Collaboration), Searches for the $Z \gamma$ decay mode of the Higgs boson and for new high-mass resonances in $p p$ collisions at $\sqrt{s}=13 \mathrm{TeV}$ with the ATLAS detector, J. High Energy Phys. 10 (2017) 112.

[35] V. Khachatryan et al. (CMS Collaboration), Search for highmass $\mathrm{Z} \gamma$ resonances in $\mathrm{e}^{+} \mathrm{e}^{-} \gamma$ and $\mu^{+} \mu^{-} \gamma$ final states in proton-proton collisions at $\sqrt{s}=8$ and $13 \mathrm{TeV}$, J. High Energy Phys. 01 (2017) 076. 